\title{
El concepto de 'perversidad' en Edgar Allan Poe. Una reflexión filosófica
}

\author{
Edgar Allan Poe's concept of wickedness. \\ A philosophical reflection
}

\section{Resumen}

En algunos cuentos como The black caty The imp of the perverse, el escritor estadounidense Edgar Allan Poe (1809-1849) trata de dar una explicación de la actitud de los personajes protagónicos haciendo uso de su concepción de la "perversidad". En los cuentos mencionados hace un esbozo de lo que podría ser una teoría de la perversidad, donde son destacables las alusiones al poco o nulo trato que filósofos y moralistas han dado a la cuestión. El presente artículo tiene como objetivo dar un primer paso en el trato filosófico de la perversidad tal y como la entiende Poe. Para ello, el escrito está dividido en dos grandes partes: la primera explorará la noción de perversidad en cuentos como The black caty The imp of the perverse, mientras que la segunda, explorará las posibles doctrinas filosóficas y teológicas que podrían emular el concepto que Poe maneja. Trataré de probar que efectivamente Poe tiene razón cuando dice que su concepto de perversidad no ha sido abordado por la filosofía y la teología del modo que él lo expone.

\section{Abstract}

In some short stories as The black cat and The imp of the perverse, the US American writer Edgar Allan Poe (1809-1849) tries to explain the attitude of the main carácter using his concept of "wickedness". Poe outlines what could be a theory of wickedness which is remarkable allusions that philosophers and moralists have given little or no treatment at all. The objective of this article is taking a first step in the philosophical treatment of how Poe understands wickedness. This paper is divided into two sections: first, it explores the notion of wickedness in short stories as The black cat and The imp of the perverse. Second, it illustrates the posible philosophical and theological doctrines that could emulate the concept that Poe uses. This paper tries to demonstrate that Poe is right when he says that his concept of wickedness has not been addressed yet in philosophy and theology.

\section{Recibido el 1 de septiembre de 2011 y aprobado el 9 de septiembre de 2011}

1 Filósofo y Ph. D en Filosofía de la Universidad Nacional de Colombia. Docente del Departamento de Filosofía de Uniminuto. Correo electrónico: jmanrrique@uniminuto.edu 


\section{¿Es usted un demonio? Soy un hombre. $Y$ por lo tanto tengo dentro de mí todos los demonios.}

G. K. Chesterton

\section{La Perversidad para Poe}

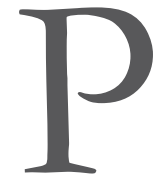

ara tratar las cosas en orden, primero expondré el concepto de perversidad en The black cat, y luego en The imp of perverse. ${ }^{2}$ También aportaré un corto resumen de los cuentos respectivos para poder dar alguna explicación respecto al papel de de la perversidad en ellos.

\section{El concepto de Perversidad en The black cat}

La historia que se desarrolla en The black cat (El gato negro) cuenta el relato de un hombre que tenía desde niño un especial afecto por los animales. En su casa, que compartía con su esposa, tenía un pez dorado, algunos conejos, un mono, un perro, algunos pájaros, y un gato negro llamado "Plutón", que era el preferido entre sus mascotas. Con el tiempo, comenzó a maltratar a sus animales e incluso a su esposa. Estando alicorado, llegó a sacarle un ojo a su gato Plutón con un corta puntas y días después lo ahorcó colgándolo de un árbol con un cordón.

Posteriormente, encontró un gato negro muy parecido a Plutón en un bar, incluso era tuerto pero no era totalmente negro, pues tenía una mancha blanca en el vientre. Se lo llevó a casa sin problemas. Ya en casa, la mancha blanca comenzó a tomar la forma de una horca y el hombre se decidió a matarlo con un hacha, pero su mujer lo contuvo, y por esta razón mató a su mujer. Decidió emparedar el cadáver de su mujer en un muro y luego buscar al gato para matarlo. Emparedó el cadáver de su esposa, pero al gato no lo encontró. Cuatro días después de estos hechos, llegó la policía a su casa debido a la desaparición de la mujer. Hicieron una minuciosa búsqueda pero no encontraron nada. El hombre estaba tan confiado de que su crimen no sería descubierto, que habló no sólo de lo fuertes que eran los muros de su casa, sino que golpeó con su bastón el muro exacto donde se encontraba el cadáver de su mujer. En ese momento se escuchó un chillido como de niño tras el muro, los policías derrumbaron el muro y notaron no sólo el cadáver de la mujer, sino al gato negro sobre su cabeza chillando. El hombre no había notado que al emparedar el cadáver había emparedado también al gato. A grandes rasgos, este es el cuento.

El papel que juega la perversidad es el de explicar (o al menos así lo parece) el por qué este hombre afable hacia los animales acaba maltratándolos, matando a su gato e incluso llega a matar a su propia esposa. La actitud bondadosa,

2 Las citas literales de estos cuentos de Poe las expondremos en inglés y en la traducción al español que de ellas hizo Julio Cortázar para Alianza Editorial. Esto para tener a la mano las palabras que usó el propio Poe y asegurarnos de que nuestra interpretación de su pensamiento es fidedigna. Es posible, claro está, que esta doble exposición revele errores en la traducción, cuestión en la que no me adentraré en este texto. 
tierna y dócil que el protagonista sostiene que le eran propias desde la infancia, y de la cual deriva su afecto a los animales, repentinamente cambió. La explicación que da Poe es la siguiente:

Our friendships lasted, in this manner, for several years, during which my general temperament and character- through the instrumentality of the Fiend Intemperance - had (I blush to confess it) experienced a radical alteration for the worse. I grew, day by day, more moody, more irritable, more regardless of the feelings of others. I suffered myself to use intemperate language to my wife. At length, I even offered her personal violence. My pets, of course, were made to feel the change in my disposition. I not only neglected, but ill-used them (Poe, 2002a, p. 16). ${ }^{3}$

El demonio de la intemperancia (The fiend intemperance) o una intemperancia demoníaca, tratada casi como una fuerza sobrenatural, es al parecer la causa de tan extraño cambio de actitud. Esta intemperancia llevará al protagonista, más adelante, a sacarle un ojo a su gato (Poe, 2002b, p. 109). Sin embargo, esta intemperancia no es la perversidad, sino más bien su vestíbulo. Veremos en qué se diferencian. Cuando el gato se recupera días después y anda tuerto de un ojo, dice el protagonista que en aquel momento sí lo invadió el espíritu de la perversidad (the spirit of Perverseness):

An then came, as if to my final and irrevocable overthrow, the spirit of PERVERSENESS. Of this spirit philosophy takes no account. Yet I am not more sure that my soul lives, than I am that perverseness is one of the primitive impulses of the human heart-one of the indivisible primary fa- culties, or sentiments, which give direction to the character of man. Who has not, a hundred times, found himself committing a vile or a stupid action for no other reason than because he knows he should not? Have we not a perpetual inclination, in the teeth of our best judgment, to violate that which is Law, merely because we understand it to be such? This spirit of perverseness, I say, came to my final overthrow. It was this unfathomable longing of the soul to vex itself- to offer violence to its own nature- to do wrong for the wrong's sake only-that urged me to continue and finally to consummate the injury I had inflicted upon the unoffending brute (Poe, 2002a, p. 18) ${ }^{4}$.

La perversidad es entonces un impulso primordial e indivisible del carácter humano. Es el impulso de cometer una cierta acción por el simple hecho de que no se debe cometer, por el simple hecho de que quebranta una ley. Es el deseo del alma de violentarse a sí misma quebrantando una ley que sabe que no debe quebrantar. Ahora, movido por la perversidad es que el protagonista decide ahorcar al gato (Poe, 2002b, p. 110), mientras que la demoníaca intemperancia sólo lo llevó a sacarle un ojo. Además, recordemos que la intemperancia por sí sola no es el móvil de la acción, recordemos que el protagonista estaba embriagado y Poe sostiene que esto ayudó a alimentar su maldad para consumar el acto de sacarle el ojo al gato (Poe, 2002b, p. 109). Encontramos así que la intemperancia y la perversidad son distintas: la intemperancia llevó a herir al gato, y eso porque estaba alimentada por el alcohol. La perversidad, en cambio, sin necesidad de alcohol, pues es un impulso humano primordial, llevó al protagonista a matar a su gato, y no sólo a herirlo.

3 "Nuestra amistad [la del protagonista con el gato] duró así [es decir, sólida] varios años, en el curso de los cuales (enrojezco al confesarlo) mi temperamento y mi carácter se alteraron radicalmente por culpa del demonio. Intemperancia. Día a día me fui volviendo más melancólico, irritable e indiferente a los sentimientos ajenos. Incluso llegué a hablar descomedidamente a mi mujer y terminé por infligirle violencias personales. Mis favoritos [los animales], claro está, sintieron igualmente el cambio de mi carácter. No sólo los descuidaba, sino que llegué a hacerles daño" (Poe, 2002b, p. 108).

4 "Y entonces, para mi caída final e irrevocable, se presentó el espíritu de la PERVERSIDAD. La filosofía no tiene en cuenta a este espíritu; y, sin embargo, tan seguro estoy de que mi alma existe como de que la perversidad es uno de los impulsos primordiales del corazón humano, una de las facultades primarias indivisibles, uno de esos sentimientos que dirigen el carácter del hombre. ¿Quién no se ha sorprendido a sí mismo cien veces en momentos en que cometía una acción tonta o malvada por la simple razón de que no debía cometerla? ¿No hay en nosotros una tendencia permanente, que enfrenta descaradamente al buen sentido, una tendencia a transgredir lo que constituye la Ley por el solo hecho de serlo?... y el insondable anhelo que tenía mi alma de vejarse a sí misma, de violentar su propia naturaleza, de hacer el mal por el mal mismo, me incitó a continuar y, finalmente a consumar el suplicio que había infligido a la inocente bestia" (Poe, 2002b, p. 110). 
Este es pues el concepto de perversidad tal como aparece en The black cat. Ciertamente el concepto sólo es enunciado y no hay mayores aclaraciones respecto a él. Debemos llegar hasta la narración de The imp of the perverse para que el concepto tome un poco más de forma por medio de algunos detalles.

\section{El concepto de Perversidad en The imp of the perverse.}

La concepción de la "perversidad" que se maneja en The imp of the perverse (El demonio de la perversidad) es algo distinta de la que nos muestra The black cat. Primero que todo, la historia o el cuento propiamente dicho de The imp of the perverse ocupa menos de la mitad de la narración, la otra gran parte no es más que una exposición filosófica de lo que Poe entiende por "perversidad". Segundo, mientras la perversidad aparece en The black cat como la explicación de las acciones homicidas del protagonista, es decir, se trata a la perversidad como una parte (o eslabón) de la historia, en The imp of the perverse la perversidad es el centro del cuento, donde la narración parece ser nada más que un caso particular de su actuación. En este apartado, resumiremos la narración propiamente dicha y luego ahondaremos en las explicaciones sobre la perversidad.

La explicación de la "perversidad" que da el protagonista parece ser la explicación de por qué está en la cárcel encadenado. Cuenta que tenía en mente un homicidio para quedarse con una herencia, pero quería que no quedara el más mínimo cabo suelto que pudiese implicarlo como el autor del asesinato. Leyó en unas memorias francesas cómo Madame Pilau había muerto por haber aspirado el humo de una vela accidentalmente envenenada. Sabía que su víctima tenía la costumbre de leer en la cama en la noche y que su habitación era pequeña y mal ventilada, razón por la cual, optó por este método. La víctima murió y las autoridades dictaminaron que había sido una muerte natural. De ese modo, el protagonista heredó el capital de la víctima y vivió tranquilo por mucho tiempo. Sin embargo, tiempo después le llegaban pensamientos al estilo "estoy a salvo, pues no sería tan estúpido como para delatarme a mí mismo". ${ }^{5}$ En ese momento su actitud cambió, y en un arrebato de "perversidad" se delató a sí mismo cuando caminaba por las calles en las que había algunas personas que al parecer lo conocían. Luego cayó al suelo desvanecido, y fue llevado a la cárcel.

Nótese que la historia difiere bastante de la de The black cat, allí, el impulso primario de la perversidad instiga al protagonista a matar a su gato y luego a su mujer. Aquí la cuestión es diferente: la perversidad instiga al protagonista a confesar su homicidio, no a cometerlo. Creo que la realización del asesinato tiene más que ver con ambiciones monetarias que con un impulso del alma. Ahora bien, vamos a retomar la explicación que se da aquí de la perversidad. Lo primero que hace Poe en el cuento es una crítica a cómo los moralistas y los frenólogos ${ }^{6}$ han pasado por alto el impulso de la perversidad.

Poe cree que la razón por la que se ignoró por tanto tiempo este impulso primitivo yace en que gran parte de la frenología y toda la metafísica fueron elaboradas a priori, de tal modo que sus abanderados imaginaron designios o propósitos divinos sobre los cuales construyeron sus sistemas (Poe, 2002b, pp. 189-190). De ese modo, a cada propósito divino le hicieron corresponder un órgano del cuerpo humano. Dios quiso que el hombre comiera, de modo que el hombre tiene un órgano de la alimentividad por medio del cual Dios lo fuerza a comer. Dios quiso que el hombre propagara su especie, de modo que dio al hombre un órgano de la amatividad, y del mismo modo hay un órgano para la combatividad,

5 "I am safe- I am safe- yes- if I be not fool enough to make open confession!" (Poe, 1978, p. 194).

6 La frenología es el estudio del carácter y las funciones intelectuales humanas, apoyado en la forma exterior del cráneo. Los médicos alemanes Gall y Sputzerheim fueron sus abanderados a principios del siglo XIX. 
la idealidad, etc. Todo órgano que represente una tendencia, un sentimiento moral o una facultad del puro intelecto era un órgano mediante el cual el Creador le establecía al hombre un designio (Poe, 2002b, p. 190). Ahora bien, Poe critica esto valiéndose precisamente de lo apriorístico de esta concepción. Sostiene que si al hombre se le mirara en sus acciones cotidianas, no sólo se le entendería mejor, sino que el impulso de la perversidad sería claramente visible:

It would have been wiser, it would have been safer, to classify (if classify we must) upon the basis of what man usually of occasionally did, and was always occasionally doing, rather than upon the basis of what we took it for granted the Deity intended him to do... Induction, a posteriori, would have brought phrenology to admit, as an innate, and primitive principle of human action, a paradoxical something, which we may call perverseness, for want of a more characteristic term. In the sense I intend, it is, in fact, a mobile without motive, a motive not motiviert. Through its promptings we act without comprehensible object; or, if it shall be understood as a contradiction in terms, we may so far modify the proposition as to say that through its promptings we act, for the reason that we should not. In theory, no reason can be more unreasonable; but, in fact, there is none more strong (Poe, 1978, pp. 186-187).

La perversidad es tratada de nuevo como en The black cat pero hay elementos nuevos. El impulso perverso es de nuevo el llevar a cabo una acción que el individuo sabe que no debe llevar a cabo. Lo nuevo es que es un impulso innato y primitivo. Con respecto a ser un impulso innato, se entiende que es un impulso con el que nacemos, y al ser primitivo, Poe quiere decir que no puede descomponerse en elementos ulteriores debido a que él mismo es elemental:
Nor will this overwhelming tendency to do wrong for the wrong's sake admit of analysis, or resolution into ulterior elements. It is a radical, a primitive impulse-elementary (Poe, 1978, p. 187). ${ }^{8}$

Poe sabe que la perversidad, tal y como la está definiendo, puede ser confundida con la combatividad de la que hablan los frenólogos, pues persistir en nuestros actos a razón de que no deberíamos realizarlos podría ser equiparado con la necesidad de la autodefensa. Poe argumenta la falsedad de esta identificación aduciendo que la combatividad, entendida como autodefensa, siempre es un acto en pro de nuestra defensa, es nuestra salvaguarda contra todo daño y su razón de ser es siempre nuestro bienestar; por el contrario, la perversidad no manifiesta, como razón de ser, nuestro bienestar, sino un sentimiento totalmente antagónico (Poe, 2002b, p. 191). Poe no aclara, hasta este punto, qué clase de sentimiento es éste, pero podemos imaginarnos el deseo del alma de vejarse a si misma del que se hablaba en The black cat, o la confesión pública del crimen del protagonista de The imp of the perverse, confesión que no contribuyó al bienestar o la salvaguarda del protagonista, sino más bien a su confinación en una cárcel.

Según Poe, el impulso de la perversidad crece hasta el deseo, el deseo hasta el anhelo, el anhelo hasta el ansia incontrolable, y ésta es consentida desafiando todas las consecuencias y con gran pesar y mortificación para quien la padece (Poe, 2002b, p. 192). Da tres ejemplos en los que obra la perversidad. El primero consiste en el deseo de un hablante de torturar a su interlocutor con circunloquios, cuando sabe el desagrado que le causa a éste. Trata de hablar con un lenguaje

7 "Hubiera sido más prudente, hubiera sido más seguro fundar nuestra clasificación (puesto que debemos hacerla) en lo que el hombre habitual u ocasionalmente hace, y en lo que siempre hace ocasionalmente, en cambio de fundarla en la hipótesis de lo que Dios pretende obligarle a hacer... La inducción a posteriori hubiera llevado a la frenología a admitir, como principio innato y primitivo de la acción humana, algo paradójico que podemos llamar perversidad a falta de un término más característico. En el sentido que le doy es, en realidad, un móvil sin motivo, un motivo no motivado. Bajo sus incitaciones actuamos sin objeto comprensible, o, si esto se considera una contradicción en los términos, podemos llegar a modificar la proposición y decir que bajo sus incitaciones actuamos por razón de que no deberíamos actuar. En teoría ninguna razón puede ser más irrazonable; pero de hecho, no hay ninguna más fuerte." (Poe, 2002b, pp. 190-191).

8 "Esta invencible tendencia a hacer el mal por el mal mismo no admitirá análisis o resolución en ulteriores elementos. Es un impulso radical, primitivo, elemental." (Poe, 2002b, p. 191). 
breve, preciso y claro, a la vez que teme y lamenta la cólera de quien lo escucha, pero al momento en que sabe que puede engendrar esa cólera con ciertos incisos y ciertos paréntesis, ese sólo pensamiento es suficiente para que su lenguaje cambie (Poe, 2002b, pp. 191-192). El segundo ejemplo consiste en tener ante nosotros una tarea que debe ser cumplida lo antes posible. Pero al saber que la demora sería ruinosa, y que la tarea debe ser acometida hoy, la dejamos para mañana, hecho que no tiene más razón que la perversidad. Al siguiente día sucede lo mismo, hasta que el conflicto interior llega hasta la última hora, en la que el "fantasma" que nos impedía acometer nuestra tarea "desaparece", nos "libera", de modo que llega el momento en que podemos acometerla, pero resulta que cuando nos es posible trabajar, ya es demasiado tarde. Ya el tiempo que teníamos para acometer la tarea ha terminado (Poe 2002b: 192).

El tercer ejemplo consiste en estar frente a un precipicio. Miramos el abismo y sentimos malestar y vértigo. A pesar de que nuestro primer impulso es retroceder ante el peligro, no lo hacemos, sino que nos invaden pensamientos como la idea de cómo serían nuestras sensaciones al caer de aquella altura, que entre otras cosas nos llevaría a una fulminante aniquilación. Es debido precisamente a que pensamos en el terrible sufrimiento y la consecuente muerte que nos quedamos, y entre más terrible se aparece a la imaginación estas cuestiones, más las deseamos. El pensamiento no nos ayuda, pues la reflexión sólo nos convencerá de que no debemos lanzarnos, lo que fortalece el impulso de hacerlo. ${ }^{9}$ Sólo podría salvarnos el que otra persona (un amigo, por ejemplo) nos detenga, o el que nos neguemos a dicho impulso de forma súbita, sin pensar, sino sencillamente echándonos atrás (Poe, 2002b, p. 193).

Por último, añade que un impulso como éste podría ser considerado como una instigación directa del demonio (archfiend), excepto porque hay casos extraordinarios en los que sirve para realizar el bien (Poe, 2002b, pp. 193-194). Es posible que esto sea alusión a la confesión pública del protagonista de The imp of the perverse, en la medida en que era justo que acabara en la cárcel.

\section{Reconstrucción del concepto de "perversidad"}

Habiendo hecho el recuento de los dos cuentos de Poe, creo que podemos enumerar lo que en términos generales entiende nuestro autor por "perversidad". Son cuatro puntos que espero que el lector tenga muy en cuenta para el examen que se llevará a cabo en la segunda parte de este escrito.

Primero, para Poe es un instinto elemental de la naturaleza humana, razón por la cual, pensadores basados en visiones a priori no tuvieron acceso a él. No es una consecuencia de ciertos factores de la naturaleza humana, sino que es constitutivo de la misma.

Segundo, es un instinto paradójico que nos hace actuar en contra de una ley, de nosotros mismos, o de ambas en el caso de que nuestro querer esté acorde con una ley, la cual puede ser moral, jurídica o religiosa. Actuamos o deseamos actuar en razón de que no deberíamos actuar. Según el propio Poe, no hay razón más irrazonable. Corrientemente hemos entendido la perversidad como una maldad extrema de alguien. No obstante, bajo este concepto tradicional podemos vislumbrar finalidades, razones, motivos o fines. El perverso corriente puede serlo porque le produce placer, porque busca mostrarse terrorífico al público, o por mero egoísmo, entre otras razones. Pero el problema con el concepto de Poe es que la perversidad de la que él trata no busca nada. Es un

9 Fortalecer el impulso quiere decir, como se dijo, convertirlo en deseo, luego en anhelo, y al final, en un ansia incontrolable 
impulso sin finalidad u objeto comprensible, la única razón para actuar de un hombre perverso en Poe es el mero hecho de que no deberíamos hacerlo, y ello implica acciones que perjudican al propio hombre perverso. Se hace el mal por el mal mismo, no porque el agente de la acción saque algún beneficio de realizarlo.

Tercero, si existiese algún objetivo o finalidad a la cual se dirige este impulso o instinto, no es otra que la autodestrucción en sentido amplio. Es el deseo del alma de "vejarse a sí misma". Es el instinto absolutamente contrario al de supervivencia. Hacemos las cosas porque no debemos hacerlas, porque hacerlas nos causaría daño. Recordemos las palabras del protagonista de The imp of the perverse: "estoy a salvo, pues no sería tan estúpido como para delatarme a mí mismo" (Poe, 1978, p. 194).

Cuarto, y esta es una característica bastante interesante: como la perversidad busca nuestro daño, es claro que no siempre este impulso está dirigido al mal de forma absoluta, o al menos de forma injusta. El caso del protagonista de The black cat es claramente el de un hombre en que el instinto lo vuelve homicida, pero el protagonista de The imp of the perverse hace confesión pública de un delito en el que no había la más mínima evidencia de que él lo hubiese cometido. En el primer cuento, la perversidad movió al protagonista al crimen, en el segundo, lo movió a confesar un crimen cuyo motivo fue la ambición. En el primer cuento, el instinto de perversidad movió al protagonista a cometer una injusticia, en el segundo, a que se hiciera justicia.

\section{Consideraciones sobre la "perversidad" en la tradición ética}

Habiendo expuesto el concepto de "perversidad" (perverseness) que sostiene Poe, vamos a dilucidar las posibles doctrinas que lo hayan tenido en cuenta. Vamos a indagar si la filosofía efectivamente se olvidó de este concepto, o si, al contrario, éste se encuentra en tradiciones éticas tan antiguas y representativas como la aristotélica y la cristiana.

\section{La perversidad y la ética aristotélica}

Dos lugares donde podríamos encontrar conceptos parecidos al descrito por Poe son la ética de Aristóteles y la descripción que da San Pablo de la dinámica interna del alma. La tradición socrático-platónica creía que la maldad en una persona era producto de su ignorancia. La virtud es un conocimiento, por tanto, la virtud es enseñable, por consiguiente, el hombre malvado no es más que un ignorante de la virtud. Aristóteles contrapone a este modelo optimista de Platón un concepto que hizo carrera en la filosofía: la $\alpha x \varrho \alpha \tau \varepsilon \iota \alpha$, (akrateia, Lit. "incontinencia")

El hombre incontinente es aquel que sabe qué debe hacer y sin embargo no puede hacerlo. Es alguien que, en principio, no es guiado por la razón sino por las pasiones; aunque su mente sepa qué es lo mejor para él, su cuerpo u otras circunstancias le hacen ceder a propuestas o impulsos que están en contra de lo que sabe que está bien hacer. Escuchemos esto de boca del mismo Aristóteles:

Podría uno preguntarse cómo es que alguien es incontinente si tiene una concepción recta. Algunos, desde luego dicen que no puede serlo si tiene conocimiento: extraño sería que, si está presente el conocimiento, lo dominara otra cosa y lo "arrastrara como a un esclavo". En efecto Sócrates combatía por completo este argumento en la idea de que no existe la incontinencia: nadie obra contra lo mejor suponiéndolo tal, sino por ignorancia. Ahora bien, este argumento está en abierta pugna con la evidencia, y es preciso seguir indagando, acerca de esta afección [...] (Aristóteles, 2004, pp. 1145b).

Aristóteles cree que la llamada "incontinencia”, es una condición de algunas personas, en contra de lo que cree la tradición socrático- 
platónica, para la cual esta condición es imposible. El hombre incontinente es aquel que sabe lo que debe hacer y no lo hace. Aristóteles usa una analogía interesante: el incontinente es como un Estado que decreta todo lo que hay que hacer y tiene leyes buenas, pero no cumple ninguna en absoluto (Aristóteles, 2004, p. 1152a).

Ahora bien. De las cuatro condiciones de Poe, vamos a examinar si las cumple. Primero, Aristóteles nunca dice que la incontinencia sea algo propio del alma humana, ni mucho menos una condición elemental. Es algo que sucede en algunas almas humanas, no en todas, pues hay personas continentes, así, podría ser una condición producida por algún desorden de las pasiones, o por falta de carácter de la persona. En su famoso tratado Acerca del Alma no hay nada que sugiera que la incontinencia es algo propio y elemental del alma humana.

Segundo, la incontinencia también consiste en actuar contra una ley que juzgamos justa y buena. Un fumador es un incontinente. Sabe que fumar causa cáncer y daño pulmonar y no obstante lo hace. Pero los personajes de los cuentos mencionados de Poe parece que tienen algo más. Ciertamente ellos también hacen lo que no deben según sus propios principios (asesinar a un ser querido, declarar contra sí mismo). Un incontinente y un perverso son idénticos en tanto cada uno de ellos rompe una ley aunque sepan que seguirla les beneficia.

Tercero, parece que el incontinente que define Aristóteles también cumple con que la finalidad u objeto de la perversidad es la autodestrucción. Un incontinente sabe que va a morir por fumar, pero lo hace. Sin embargo, la incontinencia sí tiene un objeto en Aristóteles que es el placer, y no cualquier placer, sino un tipo de placer que él lo llama vergonzoso. El placer que produce el no mentir no es un placer vergonzoso, el que produce, por ejemplo, el exceso de bebidas alcohólicas, sí lo es (Aristóteles, 2004, p. 1151b). Al tiempo, como no es un instinto elemental del alma humana, no hay algo así como un deseo del alma de "vejarse a sí misma". Sencillamente hay una especie de debilidad de la voluntad, que bien pudo ser causada por factores externos, o por no ejercitar y educar la voluntad. Lo cual nos lleva a que la condición de incontinencia puede ser curada, o al menos prevenida en las generaciones futuras, por medio de una cierta educación de la voluntad en el arte del dominio de las pasiones y de los placeres.

Por último, tenemos que la perversidad en Poe a veces sirve para el bien o para la justicia, como lo muestra la autoconfesión del protagonista de The imp of the perverse. Esto implica que la perversidad nos lleva a actuar contra ciertas leyes o principios incluso sin importar el contenido de los mismos. El incontinente tampoco actúa necesariamente contra leyes buenas, sino que puede hacerlo contra leyes perversas. El concepto de incontinencia sólo tiene en cuenta que actuemos en contra de algún principio o ley que nuestra razón sabe que está bien hacer, pero podemos creer que robar un banco está bien, pero por incontinencia no hacerlo. En este caso, la incontinencia sirvió para el bien. El caso que nos presenta Aristóteles es un pasaje de la tragedia Filoctetes del dramaturgo Sófocles. El personaje Neptólemo es persuadido por Odiseo a que mienta, cosa que Neptólemo está de acuerdo en hacer, pero al final por incontinencia no lo hace ${ }^{10}$ (Aristóteles, 2004, p. 1151b).

Podemos decir entonces que Aristóteles nos da una primera aproximación a lo que podría ser la perversidad en Poe, pero al no cumplir ni la primera, ni la tercera característica, y cumplir la cuarta sólo parcialmente, podemos decir que la incontinencia de Aristóteles no es equivalente a la perversidad en de la que nos habla Edgar Allan Poe.

10 Hago la claridad que aquí el pensamiento de Aristóteles da un viro. Él cree que Neptólemo no es un incontinente porque actuó movido por un placer bueno, el placer que produce el no mentir. Para Aristóteles sólo es incontinente el hombre cuyas acciones se producen buscando placeres vergonzosos (Aristóteles, 2004, p. 1151b). 


\section{La perversidad y su explicación en la ética cristiana}

Ciertamente Poe insiste en que esta tendencia parece ser casi una instigación directa del demonio. San Pablo ya nos había hablado de un fenómeno parecido cuando nos dice en la Carta a Romanos que el pecado habita en él, y que cuando su voluntad quiere hacer el bien es el mal el que se le presenta. Pablo describe el pecado como una fuerza incontenible que recorre los miembros de su cuerpo y lo obliga a hacer lo que él mismo aborrece. La solución de San Pablo es acogerse a la gracia obtenida por la resurrección de Cristo, ya no por la ley, pues la perversidad trae consigo el conocimiento de la ley precisamente para hacer exactamente lo que no se debe hacer, sea en contra de otros, sea en contra de uno mismo. Podríamos decir que Pablo requiere que una fuerza equivalente o más poderosa que la del pecado contrarreste las instigaciones de éste, fuerza que viene de la gracia de Cristo. Veamos esta doctrina de la propia pluma de San Pablo:

Realmente, mi proceder no lo comprendo, pues no hago lo que quiero sino lo que aborrezco. Y, si hago lo que no quiero, estoy de acuerdo con la Ley en que es buena; en realidad, ya no soy yo quien obra, sino el pecado que habita en mí. Pues bien, sé yo que nada bueno habita en mí, es decir, en mi carne; en efecto, querer el bien lo tengo a mi alcance, mas no el realizarlo, puesto que no hago el bien que quiero, sino que obro el mal que no quiero. Y, si hago lo que no quiero, no soy yo quien lo obra, sino el pecado que habita en mí. Descubro pues esta ley: aunque quiera hacer el bien, es el mal el que se me presenta. Pues me complazco en la ley de Dios según el hombre interior, pero advierto otra ley en mis miembros que lucha contra la ley de mi razón y me esclaviza a la ley del pecado que está en mis miembros. Pobre de mí, ¿quién me librará de este cuerpo que me lleva a la muerte... Así pues, soy yo mismo quien con la razón sirvo a la ley de Dios, más con la carne, a la ley del pecado (Romanos 7, 15-25).

La ley de Dios son los preceptos consignados en las tablas de los mandamientos que fueron entregados al pueblo de Israel, por medio de Moisés.
La idea de San Pablo consiste en que la Ley mosaica, por sí sola, no nos hace buenas personas. Nos indica el camino a seguir a la manera de un mapa, pero no nos impulsa a caminarlo. Yo puedo saber la ruta que debo tomar para ir a Cartagena, y no por ello tener ganas o deseos de ir a Cartagena. La Ley indica el camino pero no nos ayuda a recorrerlo. La Ley prescribió los actos que eran considerados pecados y esto hizo que conociéramos lo que era un pecado. La ley del pecado en nosotros se aprovechó de esto e hizo de nosotros algo muy parecido al incontinente aristotélico. Así, el judío de tiempos pre-cristianos, según parece seguirse de lo dicho por San Pablo, era un hombre que conocía el bien por medio de la Ley de Moisés, pero el conocimiento de la misma no le garantizaba cumplirla, pues el impulso interno del pecado no se lo permitiría.

Así, hacía falta una fuerza poderosa que contrarrestara el pecado, y nos hiciera posible ser cumplidores de los preceptos consignados en la ley. Es aquí donde San Pablo ve la necesidad que de un salvador tenían los judíos practicantes de su tiempo, es decir, de Jesucristo, el Mesías:

Por consiguiente, ninguna condenación pesa ya sobre los que están en Cristo Jesús. Porque la ley del espíritu que da la vida en Cristo Jesús te liberó de la ley del pecado y de la muerte. Pues lo que era imposible a la ley, reducida a la impotencia por la carne, Dios, habiendo enviado a su propio Hijo en una carne semejante a la del pecado, y en orden al pecado, condenó el pecado en la carne, a fin de que la justicia de la ley se cumpliera en nosotros que seguimos una conducta, no según la carne, sino según el espíritu (Romanos 8, 1-4).

No voy a entrar en la amplia discusión sobre la gracia divina, que llevó a Europa a la reforma protestante. Sólo tomo estos apartes de la doctrina de San Pablo para verificar si la tesis de Poe es algo más que un recurso literario. Enumeremos los cuatro puntos en los que enmarcamos el concepto y verifiquemos si San Pablo se acerca más a él.

Primero, la ley del pecado que habita nuestros miembros es una condición más del cuerpo que 
del alma, al tiempo que no es natural de él, sino que podemos asumir que fue impregnada a las generaciones humanas desde que Adán y Eva cometieron el pecado original, que transmitieron a todos sus descendientes.

Segundo, la ley de la carne claramente nos hace actuar contra la ley de Moisés. Por medio de ella, conocimos los actos que eran prohibidos, y la carne nos impulsó a cometerlos.

Tercero, el pecado que habita en nosotros, si bien fue adquirido, sí busca nuestra destrucción. Hacemos lo que aborrecemos, y esto claramente incluye lo que va en contra de nuestro instinto de supervivencia.

Cuarto, pienso que en el contexto de San Pablo si es muy importante el contenido de la Ley, de tal modo que no es el pecado el que nos mueve, por ejemplo, a hacer una confesión de un delito o algo por el estilo. La ley del pecado jamás nos moverá a cometer un bien, porque está en contra de la Ley de Moisés, la cual es el bien por antonomasia para la tradición judeocristiana.

De ese modo, la doctrina de San Pablo cumple satisfactoriamente el punto segundo, parcialmente los puntos primero y tercero, y no cumple definitivamente el punto cuarto. La ley del pecado de la que nos habla San Pablo en su Epistola a los Romanos no es equivalente a la "perversidad" en Edgar Allan Poe.

\section{Conclusiones}

¿Es nuevo el concepto de "perversidad" de Poe? ¿Tiene razón Poe cuando sostiene que la perversidad fue olvidada por la filosofía? Vimos que la doctrina aristotélica y la cristiana se acercaron de forma considerable; sin embargo, el concepto no fue abarcado completamente por ninguna de las tradiciones aquí tratadas. Ambas tradiciones estaban de acuerdo en que hay casos en que se realizan acciones sólo por romper una ley, es decir, estaban de acuerdo en el punto segundo de la definición de la perversidad. Con respecto al primer y tercer punto, la doctrina de Aristóteles estuvo en contra, mientras la de San Pablo estuvo parcialmente de acuerdo. Estar de acuerdo con estos puntos es aceptar que nuestra naturaleza está abocada a la autodestrucción. Nuestra vida está entrecruzada por impulsos contrarios, no hay una armonía en el alma, sino sólo conflicto, el cual es natural y no producto del mundo exterior, al tiempo, es un conflicto que no cesará. Ni Aristóteles ni San Pablo pueden aceptar completamente la idea de un componente autodestructivo al interior de nosotros mismos. Por último, Aristóteles aceptaba parcialmente el cuarto punto, San Pablo no podía en absoluto. Para Aristóteles, parecen haber ocasiones en que la incontinencia sirve al bien, en San Pablo, el pecado jamás puede ser bueno, ni servir al bien.

Tenemos entonces que Edgar Allan Poe efectivamente encontró algo nuevo, al menos con respecto a las tradiciones éticas mencionadas. Lo nuevo de su concepto tiene que ver con los puntos primero y tercero de la definición de la perversidad. La idea de que nuestra naturaleza posee un impulso maligno y autodestructivo, parece ser propia de la modernidad. Parece ser un antecedente de ideas como el famoso impulso de muerte del que nos habla Freud, o de impulsos en pugna constante como de los que nos habla Nietzsche. Si bien no es un concepto para nada alentador, podría explicar cosas como el suicidio, o lo que en su momento Michel Foucault llamó los "crímenes sin razón”. De la misma manera en que Nietzsche sostuvo que los griegos escondieron sus ideas artísticas tras las figuras de sus dioses (Nietzsche, 2007, p. 41), del mismo modo, creo que Poe escondió una idea filosófica interesante detrás de su literatura.

Al tiempo, si efectivamente tenemos razón y el concepto de "perversidad" en Poe es un concepto moral, no sólo una herramienta de deleite literario, parece necesario determinar 
cómo la filosofía moral podría abordar una realidad como ésta. El demonio de la perversidad no parece ser una condición de ciertos individuos como la esquizofrenia. No parece haber factores desencadenantes claros, sino que Poe cree que es una condición humana sin más, todos estamos, por consiguiente, proclives a ser perversos en algún momento de nuestra vida. Hacen falta nuevas perspectivas éticas que aborden este interesante y aterrador concepto.

\section{Referencias bibliográficas}

Aristóteles. (2004). Ética a Nicómaco. Traducción de José Luis Calvo Martínez. Madrid: Alianza Editorial.

Biblia de Jerusalén. (2000). Bilbao: Desclée de Brouwer.

Nietzsche, F. (2007). El nacimiento de la tragedia. Traducción de Andrés Sánchez Pascual. Madrid: Alianza Editorial.
Poe, E. A. (1978). The imp of the perverse. En: Poe, E. A. Eight Tales of Terror. New York: Scholastic Inc. - (2002a). The black cat and other Tales. Bogotá: The English Bookmarks. Panamericana Editorial. (2002b). Cuentos, 1. Prólogo, traducción y notas de Julio Cortázar. Madrid: Alianza Editorial. 